\begin{tabular}{|c|l|}
\hline Title & Ergothioneine protects Streptomyces coelicolor A 3(2) from oxidative stresses \\
\hline Author(s) & Nakajima, Shunsuke; Satoh, Y asuharu; Y anashima, Kentaro; Matsui, Tomomi; Dairi, Tohru \\
\hline Citation & Journal of bioscience and bioengineering, 120(3), 294.298 \\
\hline https://doi.org/10.1016/.jbiosc.2015.01.013 \\
\hline Issue Date & 2015-09 \\
\hline Doc URL & http://hdl.handle.net/2115/62799 \\
\hline Rights & ○ 2015 The Society for Biotechnology, Japan \\
\hline Rights(URL) & http://creativecommons.org/icenses/by-nc-nd/4.0/ \\
\hline Type & article (author version) \\
\hline File Information & Satoh-JBB120(3).pdf \\
\hline
\end{tabular}

Instructions for use 


\section{Ergothioneine protects Streptomyces coelicolor A3(2) from oxidative stresses}

3 Shunsuke Nakajima ${ }^{1}$, Yasuharu Satoh ${ }^{1}$, Kentaro Yanashima ${ }^{2}$, Tomomi Matsui ${ }^{1}$, and Tohru Dairi ${ }^{1}$ *

$5{ }^{1}$ Graduate School of Engineering, Hokkaido University, Hokkaido 060-8628, Japan

$6 \quad{ }^{2}$ Kyowa Hakko Bio Co. Ltd., Chiyoda-ku, Tokyo 100-8185, Japan

*Corresponding author, Graduate School of Engineering, Hokkaido University, Hokkaido

10 060-8628, Japan. Tel. +81-11-706-7815; Fax. +81-11-706-7118; E-mail: dairi@eng.hokudai.ac.jp

12 Running title: Function of ergothioneine in a Streptomyces strain

13

14 Key words: ergothioneine; mycothiol; Streptomyces coelicolor; biological function

15

16

17

18

19

20 
1 Thiol compounds with low-molecular weight, such as glutathione, mycothiol (MSH),

2 bacillithiol, and ergothioneine (ERG), are known to protect microorganisms from oxidative

3 stresses. Mycobacteria and actinobacteria utilize both MSH and ERG. The biological functions of

4 MSH in mycobacteria have been extensively studied by genetic and biochemical studies, which

5 have suggested it has critical roles for detoxification in cells. In contrast, the biological functions

6 of ERG remain ambiguous because its biosynthetic genes were only recently identified in

$7 \quad$ Mycobacterium avium. In this study, we constructed mutants of Streptomyces coelicolor A3(2), in

8 which either the MSH or ERG biosynthetic gene was disrupted, and examined their phenotypes.

9 A mshC (SCO1663)-disruptant completely lost MSH productivity. In contrast, a disruptant of the

10 egtA gene (SCO0910) encoding $\gamma$-glutamyl-cysteine synthetase unexpectedly retained reduced

11 productivity of ERG, probably because of the use of L-cysteine instead of $\gamma$-glutamyl-cysteine.

12 Both disruptants showed delayed growth at the late logarithmic phase and were more susceptible

13 to hydrogen peroxide and cumene hydroperoxide than the parental strain. Interestingly, the

14 ERG-disruptant, which still kept reduced ERG productivity, was more susceptible. Furthermore,

15 the ERG-disruptant accumulated 5-fold more MSH than the parental strain. In contrast, the

16 amount of ERG was almost the same between the MSH-disruptant and the parental strain. Taken

17 together, our results suggest that ERG is more important than MSH in S. coelicolor A3(2).

23 Microorganisms utilize several thiol compounds with low-molecular weight to maintain a

24 reducing environment (1). Glutathione is a representative compound found in gram-negative 25 microorganisms and glutathione reductases reduce oxidized glutathione for recycling. 
1 Glutathione S-transferase and glutaredoxin also participate in the reduction of the oxidative state

2 using glutathione (1). In E. coli, glutathione and glutathione reductase are not essential for growth

3 but a glutathione-disruptant became more susceptible to chemical toxins and oxygen than the

4 parental strain. Moreover, disruption of all of the genes encoding glutathione reductase and

5 glutaredoxin was lethal (1).

6 In contrast to gram negative bacteria, gram-positive microorganisms do not use glutathione 7 to maintain reducing conditions but make use of mycothiol (MSH), bacillithiol, and 8 ergothioneine (ERG) compounds (1). MSH is widespread in actinobacteria. It was first identified 9 in a cell extract of a Streptomyces strain in 1993 (2) and then its structure was determined $(3,4)$. 10 Recently, the biosynthetic pathway of MSH was established and the functions of four 11 biosynthetic genes were characterized (5,6). The biological function of MSH is essentially the 12 same as that of glutathione, and mycothione (oxidized form of MSH) reductase catalyzes the 13 reduction of the disulfide form into MSH (1). In Mycobacterium tuberculosis, MSH is essential 14 for in vitro growth (5). In contrast, MSH is not essential in Mycobacterium smegmatis but 15 MSH-disruptants showed increased sensitivity to oxidative stress, alkylating agents, and some 16 antibiotics (1). In Firmicutes, bacillithiol, the structure of which is similar to that of MSH, is 17 utilized (7). By taking advantage of the structural similarities, the biosynthetic genes of 18 bacillithiol were identified (8). A bacillithiol-disruptant of Bacillus subtilis showed the same 19 phenotype as glutathione- and MSH-disruptants (1).

20 In mycobacteria, actinobacteria, and certain fungi, ERG is also utilized together with MSH.

21 In the late 1960s, the building blocks of ERG were identified to be histidine, cysteine, and 22 methionine $(9,10)$. Until recently, there were no reports on the biosynthetic genes and enzymes of 23 ERG. However, five genes, egtA to E, in Mycobacterium avium were recently confirmed to be 24 responsible for ERG biosynthesis (11). As for the biological function of ERG, a MSH-disrupted 25 M. smegmatis mutant was reported to accumulate 2 to 26-fold higher ERG levels $(12,13)$, 
1 suggesting that ERG has the same function as MSH. However, the detailed biological function of

2 ERG in bacterial cells is not well understood because it has only been a short period since the

3 biosynthetic genes were discovered. Recently, a MSH-disruptant, an ERG-disruptant, and a

4 disruptant of both were constructed in M. smegmatis and both single disruptants were shown to

5 be susceptible to peroxide (13). The double-disruptant was also significantly more susceptible to

6 peroxide than either of the single disruptants.

$7 \quad$ Streptomyces strains are industrially very important microorganisms because they produce a

8 variety of anti-bacterial compounds that can be candidates for antibiotic development. Recently,

9 different classes of antibiotics were shown to contribute to killing bacteria by generating

10 deleterious reactive oxygen species including hydrogen peroxide, in addition to their intrinsic

11 drug-target interactions (14). This result suggests that antibiotics induce redox alterations that

12 lead to cellular damage even in their producers. In this study, we used Streptomyces coelicolor

$13 \mathrm{~A} 3(2)$ as a model strain and constructed mutants, in which either the MSH or ERG biosynthetic

14 gene was disrupted, to investigate the biological functions of both of these thiol compounds. Both

15 the MSH-disrupted and ERG-disrupted S. coelicolor A3(2) were more susceptible to hydrogen

16 peroxide than the parental strain and showed different phenotypes from those of $M$. smegmatis

17 mutants. Furthermore, our results suggested ERG was more important than MSH in S. coelicolor $18 \mathrm{~A} 3(2)$

\section{Materials and Methods}

22 Chemicals ERG was obtained from Cosmo Bio Co. Ltd (Tokyo, Japan). Cumene hydroperoxide,

23 diamide, and monobromobimane $(\mathrm{mBBr})$ were purchased from Sigma-Aldrich Japan (Tokyo,

24 Japan). All other chemicals were analytical grade. 
1 DNA isolation and manipulation Plasmids from E. coli were prepared using a Qiagen plasmid

2 kit (QIAGEN Japan, Tokyo). All restriction enzymes, T4 DNA ligase, and calf intestinal alkaline

3 phosphatase were obtained from Toyobo (Osaka, Japan). Transformations of E. coli and $S$.

4 coelicolor A3(2) with plasmid DNA were performed by electroporation under standard conditions

5 using a BTX ECM 600 electroporation system (Biotechnologies and Experimental Research, Inc.,

6 San Diego, CA) and by the protoplast-polyethylene glycol assisted method (15). Other general

7 procedures were performed as described by Maniatis et al. (16).

9 Preparation of SCO0910 recombinant enzyme The SCO0910 gene was amplified by PCR 10 using gene-specific primers, P1 and P2 (Table S1). After subcloning and sequence confirmation, 11 1.3-kb fragments obtained by NdeI and HindIII digestion were ligated to the corresponding site of 12 pMAL-c5X (New England Biolabs Japan, Tokyo). The constructed plasmid was designated 13 pMAL-SCO0910. E. coli XL-1 blue harboring pMAL-c5X (control) and pMAL-SCO0910 were 14 separately grown in Luria-broth supplemented with $100 \mu \mathrm{g} / \mathrm{mL}$ ampicillin. The strain was grown 15 at $37^{\circ} \mathrm{C}$ until $\mathrm{OD}_{600}$ reached 0.5 and then isopropyl $\beta$-D-thiogalactopyranoside $(0.5 \mathrm{mM})$ was 16 added to the culture, followed by additional cultivation at $18^{\circ} \mathrm{C}$ for $18 \mathrm{~h}$. After the cells were 17 harvested and washed once with chilled buffer A (50 mM Tris-HCl, $500 \mathrm{mM} \mathrm{NaCl,} \mathrm{pH} \mathrm{7.5),} \mathrm{they}$ 18 were suspended in the same buffer and then disrupted by sonication. The debris was removed by 19 centrifugation, the supernatant was applied to amylose resin (New England Biolabs) and the 20 recombinant enzyme was purified according to the manufacturer's instructions. The eluent was 21 desalted and concentrated using an Amicon apparatus (Merck Japan, Tokyo, Japan). After the 22 purity of the recombinant enzyme was checked by SDS-PAGE, the enzyme was used for in vitro 23 assay.

24 The standard assay mixture for SCO0910 contained, in a final volume of $200 \mu \mathrm{L}, 12.5 \mathrm{mM}$ 25 of L-glutamate, $12.5 \mathrm{mM}$ of L-cysteine, $12.5 \mathrm{mM}$ of ATP, $12.5 \mathrm{mM}$ of $\mathrm{MgSO}_{4}, 50 \mathrm{mM}$ Tris- $\mathrm{HCl}$ 
1 (pH 8.0), and a suitable amount of recombinant SCO0910. After the mixture was incubated at

$237^{\circ} \mathrm{C}$ overnight, the products were analyzed by LC-ESI-MS (Waters ACQUITY UPLC equipped

3 with a SQ Detector2; Nihon Waters, Tokyo, Japan). The analytical conditions were as follows:

4 Merck SeQuant $^{\mathrm{TM}}$ ZIC-HILIC PEEK HPLC column $(2.1 \times 150 \mathrm{~mm}, 5 \mu \mathrm{m})$; column temperature,

$535^{\circ} \mathrm{C}$; detection, positive mode; mobile phase, $20 \mathrm{mM}$ ammonium acetate (pH 6.8):acetonitrile =

$6 \quad$ 15:85 for $60 \mathrm{~min}$; flow rate, $0.3 \mathrm{ml} / \mathrm{min}$; cone voltage, $20 \mathrm{~V}$.

8 Disruption of the SCO0910 and SCO1663 genes The SCO0910 and SCO1663 genes, which

9 encode EgtA (EC 6.3.2.2) and MshC (EC 6.3.1.13), respectively, were disrupted by

10 double-crossover homologous recombination. Two DNA fragments carrying the upstream and

11 downstream regions of each of the target genes $(2 \mathrm{~Kb})$ were amplified with a set of primers

12 (listed in Table S1 and Fig. S1) by PCR. After sequence confirmation, these two fragments were

13 inserted into the appropriate restriction sites of pUC18 in the same direction as in the genomic

14 region. DNA fragments carrying the thiostrepton resistance gene (Table S1 and Fig. S1) were

15 then inserted between the above-mentioned two fragments. The constructed plasmids were

16 digested with ScaI and used to transform S. coelicolor A3(2). Disruption of the genes in

17 thiostrepton resistant colonies was confirmed by PCR analysis (Fig. S1). Production of ERG and

18 MSH was confirmed by LC-ESI-MS analysis as described below.

20 Growth of the disruptants To examine the effects of ERG- or MSH-deficiency on growth, the 21 disruptants were cultivated with $3 \mathrm{ml}$ of SK No. 2 medium (17) in a test tube for 3 days at $30^{\circ} \mathrm{C}$.

22 An aliquot of the culture $(0.5 \mathrm{~mL})$ was inoculated into $50 \mathrm{ml}$ of fresh SK No. 2 medium in a 200

$23 \mathrm{ml}$ Erlenmeyer flask and cultured for $50 \mathrm{~h}$. Samples of the cell culture were collected over a time

24 course and growth was measured by dry cell weight $(\mathrm{g} / \mathrm{L})$. 
1 Analysis of productivities of thiol compounds The disruptants and the parental strain were

2 cultivated in the same manner as described above. Mycelia were collected at late logarithmic

3 phase when the dry cell weight reached about $5 \mathrm{~g} / \mathrm{L}$. Since both of the disruptants grew slower

4 than the parental strain, the former and the latter were collected after $48 \mathrm{~h}$ and $36 \mathrm{~h}$ of cultivation,

5 respectively. After centrifugation, both the mycelia and the supernatant were used for

6 measurement of thiol compounds. For the analysis of intracellular compounds, the mycelia

7 collected from $20 \mathrm{~mL}$ of culture broth were washed with $10 \mathrm{ml}$ of ultrapure water and then 200

$8 \mathrm{mg}$ of the collected cells were suspended in $500 \mu \mathrm{L}$ of buffer A containing $50 \%$ acetonitrile, 2

$9 \mathrm{mM} \mathrm{mBBr}$, and $20 \mathrm{mM}$ Tris $\mathrm{HCl}(\mathrm{pH}$ 8.0). The mycelia were disrupted with a sonicator (Branson

$10450 \mathrm{D}$, Emerson CT, USA) and incubated at $60^{\circ} \mathrm{C}$ for $15 \mathrm{~min}$ to produce $\mathrm{mBBr}$-derivatives (18).

11 After the reaction, $25 \mu \mathrm{l}$ of $5 \mathrm{M}$ methanesulfonic acid solution was added and the supernatants

12 obtained by centrifugation were analyzed by LC-ESI-MS after appropriate dilution. For the

13 analysis of secreted compounds, the supernatant was filtrated and concentrated by free-drying.

14 The dried samples were dissolved with buffer A. The subsequent protocol was the same as that

15 for intracellular compounds. LC-ESI-MS analytical conditions were as follows: InertSustain C18

16 HP column $(150 \mathrm{~mm} \times 2.1 \mathrm{~mm}$ ID, $3 \mu \mathrm{m}$; GL Sciences Inc., Tokyo, Japan); column temperature,

$1740^{\circ} \mathrm{C}$; detection, positive mode; mobile phase, $0.05 \%$ trifluoroacetic acid solution:methanol =

$1890: 10$ at $3 \mathrm{~min}$, and a linear gradient to 15:85 for an additional $30 \mathrm{~min}$; flow rate, $0.3 \mathrm{ml} / \mathrm{min}$;

19 cone voltage, $40 \mathrm{~V}$. The amounts of thiol compounds were normalized by cell growth (dry cell

20 weight). The productivities of the thiol compounds except for MSH were calculated with a

21 standard curve. MSH productivity was shown as relative values; the amount of MSH produced by

22 the parental strain was defined as 1 since MSH was not commercially available.

24 Examination of sensitivity to peroxide The sensitivity of the ERG- and MSH-disruptants

25 against peroxides was tested by a paper disk-agar diffusion assay, which is based on the 
1 phenomenon that toxic compounds will diffuse from a paper disc into an agar medium containing

2 test microorganisms and form a growth-inhibitory zone. The two disruptants and the parental

3 strain (control) were cultivated in the same manner as described above. Molten soft agar

4 containing the test microorganisms was spread on ATCC5 plates $(0.2 \%$ starch, $0.1 \%$ glucose,

$5 \quad 0.1 \%$ Bacto yeast extract, $0.1 \%$ fish meat extract, $0.01 \% \mathrm{FeSO}_{4}, 2 \%$ agar, $\mathrm{pH}$ 7.2). Paper disks

6 containing various concentrations of peroxides were put onto the lawn of cells. After incubation

7 for 2 days, the size of the halo was measured and the minimum inhibition concentration (MIC)

8 was determined.

10 Examination of organic hydroperoxide resistance (Ohr) protein production Induction of the

11 organic hydroperoxide resistance protein (SCO2396, SCO2986, and SCO7111) was examined by

12 SDS-PAGE analysis. Mycelia of the disruptants and the parental strain, which were cultivated by

13 the method described above, were harvested by centrifugation. After being suspended in $50 \mathrm{mM}$

14 of sodium-phosphate buffer ( $\mathrm{pH}$ 8.0) containing $300 \mathrm{mM}$ of sodium chloride, the cells were

15 disrupted by ultrasonic oscillation at $4^{\circ} \mathrm{C}$ for $5 \mathrm{~min}$. Supernatants obtained by centrifugation of

16 the cell lysates were analyzed by SDS-PAGE on 15\% gels. Protein concentration was determined

17 by a protein-dye standard assay (Bio-Rad) using bovine serum albumin as a standard.

\section{Results and Discussion}

22 SCO0910 encodes $\gamma$-glutamyl-cysteine synthetase Five genes, egtA to egtE, in Mycobacterium

23 avium were recently confirmed to be responsible for ERG biosynthesis (11) (Fig. 1). We searched

24 for orthologs of these genes in the genome database of S. coelicolor A3(2) and identified

25 SCO0910 to SCO0913 as genes corresponding to egtA to egtD in M. avium. SCO1921, which was 
1 located outside of the gene cluster, was suggested to be an ortholog of egtE. To determine

2 whether these genes indeed encoded the ERG biosynthetic enzymes, the function of SCO0910,

3 which possibly encoded $\gamma$-glutamyl-cysteine synthetase (EgtA), was examined with a

4 recombinant enzyme. Maltose binding protein-fused recombinant SCO0910 (89.6 kDa) was

5 successfully expressed in a soluble form and the purified enzyme was subjected to SDS-PAGE

6 analysis to confirm its molecular size and purity (Fig. 2A).

$7 \quad$ The catalytic activity was then examined. The purified recombinant SCO0910 was incubated

8 with L-glutamate and L-cysteine in the presence of ATP, and the reaction product was analyzed by

9 LC-ESI-MS. As shown in Fig. 2B, the formation of $\gamma$-glutamyl-cysteine was specifically

10 detected.

12 Construction of ERG- and MSH- disruptants To investigate the biological functions of ERG

13 and MSH in S. coelicolor A3(2), we constructed two types of mutants, in which the respective

14 biosynthetic genes were disrupted by homologous recombination. In the ERG-disruptant, we

15 disrupted the SCO0910 gene by homologous recombination since SCO0910 was confirmed to be

$16 \gamma$-glutamyl-cysteine synthetase as described above. A disruptant, in which the SCO0910 gene was

17 replaced with the thiostrepton resistance gene, was constructed and the intended disruption was

18 confirmed by PCR (Fig. S1 and Table S1). After cultivation of the disputant, we examined the

19 production of ERG. Since a significant portion of ERG was recently reported to be secreted into

20 the culture broth (13), both secreted and intracellular ERG were measured. Unexpectedly, the

21 disruptant still retained ERG productivity. Intracellular and secreted ERG levels were estimated

22 to be $20 \%$ and $85 \%$ of those of the parental strain (Table 1 and Fig. S2). In the biosynthesis of

23 ovothiol, which has similar structure and function to ERG (19), OvoA was shown to catalyze the

24 incorporation reaction of thiol with L-cysteine instead of $\gamma$-glutamyl-cysteine (20). In $S$.

25 coelicolor A3(2), EgtB corresponding to OvoA, might utilize L-cysteine in a similar manner to 
OvoA.

In the case of the MSH-disruptant, SCO4204, SCO1663, and SCO4151 encoding MshA, C,

3 and D, respectively, were previously confirmed to be essential for MSH biosynthesis (Fig. S3) by

4 a gene-disruption experiment (21). We therefore disrupted the SCO1663 gene encoding mshC by

5 the same method as described above. We successfully obtained the intended disruptant, (Table S1

6 and Fig. S1), suggesting that MSH is not essential in S. coelicolor A3(2) similar to M. smegmatis

$7 \quad(6,12)$, and the absence of MSH production was confirmed by LC-ESI-MS as previously reported

8 (21) (Fig. S2).

10 Effects of ERG or MSH-deficiency on growth We first examined the effects of ERG- or

11 MSH-deficiency on growth. In the case of $M$. smegmatis, the $\Delta$ egtD and $\Delta m s h A$ disruptants were

12 shown to grow normally (13) and slightly slower than the parental strain on agar plates (6),

13 respectively. The $\Delta S C 00910$ disruptant (ERG-disruptant), $\Delta S C 01663$ disruptant

14 (MSH-disruptant) and parental strain were cultivated in liquid medium for $50 \mathrm{~h}$ and cell growth

15 was measured by dry cell weight. No differences were observed until mid-logarithmic phase but

16 the growth of both disruptants was delayed at late-logarithmic phase (Fig. 3) in contrast to the $M$.

17 smegmatis mutant. These results suggested that both MSH and ERG are necessary for normal

18 growth at the late-logarithmic phase. In particular, the delayed growth of the ERG-disruptant,

19 which still produced a reduced amount of ERG, suggested that the ERG level for normal growth

20 at this stage was critical. ERG might play a critical role for differentiation at the late-logarithmic

21 phase such as secondary metabolite productions and spore formations.

23 Productivity of thiol compounds in disruptants Previously, $\Delta m s h A$ disruptants of $M$.

24 smegmatis were shown to produce 2 to 26-fold more ERG than the parental strain, probably to 25 compensate for the loss of MSH $(12,13)$. We therefore examined whether similar phenomena 
1 were observed in the ERG- and MSH-disruptants of S. coelicolor A3(2). We measured the

2 amounts of ERG and MSH at the late-logarithmic phase since both compounds were suggested to

3 be required for normal growth at this stage as described above. The MSH-disruptant produced

4 almost the same amount of ERG as the parental strain, differing from the above-mentioned

$5 \Delta$ sshA disruptants of $M$. smegmatis (Table 1). On the other hand, the ERG-disruptant

6 accumulated 5-fold more MSH, despite the fact that the disruptant still retained ERG productivity.

7 This phenomenon was also quite different from that of a $\Delta \operatorname{erg} D$ disruptant of $M$. smegmatis,

8 which accumulated the same amount of MSH as the parental strain (13).

$9 \quad$ We next measured the amounts of L-cysteine and $\gamma$-glutamyl-cysteine to examine whether 10 these compounds compensated for the loss of MSH and ERG. In the case of the MSH-disruptant, 11 both compounds increased approximately three times compared with the parental strain. On the 12 other hand, the ERG-disruptant, in which the $\gamma$-glutamyl-cysteine synthetase gene was disrupted 13 and no $\gamma$-glutamyl-cysteine was produced (Table 1), accumulated four times as much L-cysteine.

14 These results were different from those of the MSH-disruptant of M. smegmatis (mshA(G32D) 15 mutant), which accumulated a normal amount of L-cysteine (1).

17 Production of organic hydroperoxide resistance protein (Ohr) In $M$. smegmatis, a $\Delta m s h$

18 disruptant was shown to overproduce Ohr protein, which participates in resistance to cumene 19 hydroperoxide and isoniazid (12). Since S. coelicolor A3(2) was suggested to possess three 20 orthologs of Ohr (SCO2396, SCO2986, and SCO7111) by a Blast search, we examined whether 21 these proteins were also overproduced in the ERG- and MSH-disruptants. However, no obvious 22 proteins with the molecular size $(15 \mathrm{kDa})$ of Ohr were overproduced in either disruptant, though 23 the patterns of some proteins were slightly different between them (Fig. S4). 
1 Sensitivity to various stresses Both $\Delta m s h$ and $\Delta e g t D$ disruptants of $M$. smegmatis were reported

2 to have increased susceptibility to agents generating reactive oxygen species $(1,12,13)$. To study

3 the biological functions of both compounds in S. coelicolor, A3(2), a paper disk-agar diffusion

4 assay was employed. We used hydrogen peroxide, cumene hydroperoxide, and diamide as agents

5 generating reactive oxygen species. As shown in Table 2, both the ERG- and MSH-disruptants

6 were more sensitive to hydrogen peroxide and cumene hydroperoxide than the parental strain.

7 The ERG-disruptant, which still produced a reduced level of ERG, was unexpectedly more

8 susceptible to both compounds, especially to hydrogen peroxide. The amounts of intracellular

9 ERG might be critical for the protection from hydrogen peroxide. Otherwise, the increased

10 amounts of L-cysteine in the ERG-disruptant (Table 1) might become toxic in the disruptants

11 since L-cysteine was known to show cytotoxicity at even low concentrations in E. coli (22).

12 In conclusion, we constructed disruptants of Streptomyces coelicolor A3(2), in which either

13 the MSH or ERG biosynthetic gene was disrupted, and examined their phenotypes to determine

14 the physiological roles of these thiol compounds. The mshC (SCO1663)-disruptant completely

15 lost MSH productivity. In contrast, the disruptant of the egtA gene (SCO0910) encoding $16 \gamma$-glutamyl-cysteine synthetase unexpectedly kept reduced productivity of ERG. Both the ERG17 and MSH-disruptants showed delayed growth at late-logarithmic phase and were susceptible to 18 hydrogen peroxide and cumene hydroperoxide. These results suggested that both ERG and MSH 19 protect S. coelicolor A3(2) against oxidative stress. In particular, the ERG-disruptant, which still 20 retained reduced productivity of ERG, produced 5-fold more MSH and the MSH-disruptant 21 produced almost the same amount of ERG as the parental strain. Furthermore, the 22 ERG-disruptant was more sensitive to hydrogen peroxide than the MSH-disruptant. Together,

23 these results suggest that ERG and MSH play a critical role in S. coelicolor A3(2) and M. 24 smegmatis, respectively.

25 Supplementary data related to this article can be found at http://

26 dx.doi.org/10.1016/j.jbiosc.2015.01.013. 


\section{Acknowledgments}

3 This work was supported by Grants-in-Aid for Scientific Research (24651235) to T. Dairi from

4 the Japan Society for the Promotion of Science (JSPS). 
References

3 1. Fahey, R.C.: Glutathione analogs in prokaryotes. Biochim. Biophys. Acta., 1830, $4 \quad 3182-3198$ (2013).

5 2. Newton, G.L., Fahey, R.C., Cohen G, and Aharonowitz, Y.: Low molecular weight thiols in streptomycetes and their potential role as antioxidants. J. Bacteriol., 175, 27342742 (1993).

3. Sakuda, S., Zhou, Z.Y., and Yamada, Y.: Structure of a novel disulfide of 2-(N-acetylcysteinyl)amido-2-deoxy- $\alpha$-D-glucopyran-osyl-myo-inositol Produced by Streptomyces sp. Biosci. Biotech. Biochem., 58, 1347-1348 (1994).

4. Newton, G.L., Bewley, C.A., Dwyer, T.J., Horn, R., Aharonowitz, Y., Cohen, G., Davies, J., Faulkner, D.J., and Fahey, R.C.: The structure of U17 isolated from Streptomyces clavuligerus and its properties as an antioxidant thiol. Eur. J. Biochem., 230, 821-825 (1995).

5. Newton, G.L., Buchmeier, N., and Fahey, R.C.: Biosynthesis and functions of mycothiol, the unique protective thiol of Actinobacteria. Microbiol. Mol. Biol. Rev., 72, 471-494 (2008).

6. Xu, X., Vilchèze, C., Av-Gay, Y., Go'mez-Velasco, A., and Jacobs, Jr., W.R.: Precise Null deletion mutations of the mycothiol synthesis genes reveal their role in isoniazid and ethionamide resistance in Mycobacterium smegmatis, Antimicrob. Agents Chemother., 55, 3133-3139 (2011).

7. Newton, G.L,. Rawat, M., La Clair, J.J., Jothivasan, V.K., Budiarto, T., Hamilton, C.J., Claiborne, A., Helmann, J.D., and Fahey, R.C.: Bacillithiol is an antioxidant thiol produced in Bacilli. Nat. Chem. Biol., 5, 625-627 (2009). 
Claiborne, A., Fahey, R.C., and Helmann, J.D.: Biosynthesis and functions of bacillithiol, a major low-molecular-weight thiol in Bacilli. Proc. Natl. Acad. Sci. USA, 107, 6482-6486 (2010).

9. Genghof, D.S., and Van Damme, O.: Biosynthesis of ergothioneine and hercynine by mycobacteria. J. Bacteriol., 87, 852-862 (1964).

10. Genghof, D.S., and Van Damme, O.:) Biosynthesis of ergothioneine from endogenous hercynine in Mycobacterium smegmatis. J. Bacteriol., 95, 340-344 (1968).

11. Seebeck, F.P.: In vitro reconstitution of Mycobacterial ergothioneine biosynthesis. J. Am. Chem. Soc., 132, 6632-6633 (2010).

12. Ta, P., Buchmeier, N., Newton, G.L., Rawat, M., and Fahey, R.C.: Organic

14. Dwyer, D.J., Belenky, P.A., Yang, J.H., MacDonald, I.C., Martell, J.D., Takahashi, N., Chan, C.T., Lobritz, M.A., Braff, D., Schwarz, E.G., and other 9 authors: Antibiotics induce redox-related physiological alterations as part of their lethality. Proc. Natl. Acad. Sci. USA, 111, E2100-E2109, doi: 10.1073/pnas.1401876111 (2014).

15. Hopwood, D.A., Bibb, M.J., Chater, K.F., Kieser, T., Bruton, C.J., Kieser, H.M., Lydiate, D.J., Smith, C.P., Ward, J.M., and Schremp, H.: Gene manipulation of Streptomyces, a laboratory manual. The John Innes Foundation, Norwich, UK (1985).

16. Maniatis, T., Fritsch, E.F., and Sambrook, J.: Molecular cloning: a laboratory manual, Cold Spring Harbor Laboratory, Cold Spring Harbor, New York (1989).

17. Dairi, T., and Hasegawa, M.: Common biosynthetic feature of fortimicin-group 
antibiotics. J. Antibiot (Tokyo), 42, 934-943 (1989).

18. Fahey, R.C. and Newton, G.L.: Determination of low-molecular-weight thiols using monobromobimane fluorescent labeling and high-performance liquid chromatography. Methods Enzymol., 143, 85-96 (1987).

19. Spies, H.S., and Steenkamp, D.J.: Thiols of intracellular pathogens. Identification of ovothiol A in Leishmania donovani and structural analysis of a novel thiol from Mycobacterium bovis. Eur. J. Biochem., 224, 203-213 (1994).

20. Hu, W., Song, H., Sae Her, A., Bak, D.W., Naowarojna, N., Elliott, S.J., Qin, L., Chen, X., and Liu, P.: .Bioinformatic and biochemical characterizations of $\mathrm{C}-\mathrm{S}$ bond formation and cleavage enzymes in the fungus Neurospora crassa Ergothioneine Biosynthetic Pathway. Org. Lett., 16, 5382-5385 (2014).

12 21. Park, J.H., Cha, C.J., and Roe, J.H.: Identification of genes for mycothiol biosynthesis in Streptomyces coelicolor A3(2). J. Microbiol., 44, 121-125 (2006). 
1 TABLE 1. Secreted and intracellular thiol compounds of the parental strain, ERG-disruptant, 2 and MSH-disruptant.

3

4

\begin{tabular}{|c|c|c|c|c|c|}
\hline \multicolumn{2}{|c|}{ WT } & \multicolumn{2}{|c|}{ ERG- disruptant } & \multicolumn{2}{|c|}{ MSH- disruptant } \\
\hline intracellular & secreted & intracellular & secreted & intracellular & secreted \\
\hline
\end{tabular}

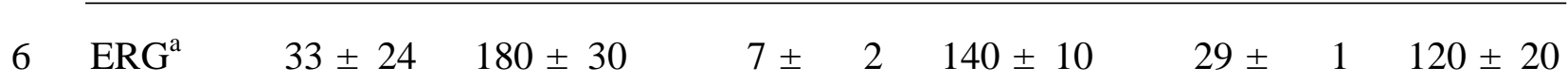

$7 \quad \mathrm{Cys}^{\mathrm{a}} \quad 140 \pm 70 \quad 150 \pm 10 \quad 590 \pm 130 \quad 280 \pm 30 \quad 450 \pm 120 \quad 440 \pm 30$

$\begin{array}{llllllll}8 & \gamma-\text { GC }^{\mathrm{a}} & 12 \pm 2 & \mathrm{ND} & \mathrm{ND} & \mathrm{ND} & 35 \pm 10 & \text { ND }\end{array}$

$\begin{array}{llllllll}9 & \mathrm{MSH}^{\mathrm{b}} & 1 & \mathrm{ND} & 5.13 & \mathrm{ND} & \mathrm{ND} & \text { ND }\end{array}$

10

$11 \quad$ a $n$ nM/DCW (g).

12 b; relative values; the amount of MSH produced by the parental strain was defined as 1.

13 ND, Not detected.

14 
1 TABLE 2. Minimum inhibition concentration (mM) of hydrogen peroxide, cumene 2 hydroperoxide $(\mathrm{CuOOH})$, and diamide against the parental strain, ERG-disruptant, and 3 MSH-disruptant.

4

\begin{tabular}{llllrc}
5 & & WT & & ERG- disruptant & MSH- disruptant \\
\cline { 2 - 7 } 6 & $\mathrm{H}_{2} \mathrm{O}_{2}$ & 13.8 & \pm 1.1 & $3.5 \pm 0.5$ & $10.1 \pm 2.2$ \\
7 & $\mathrm{CuOOH}$ & 14.0 & \pm 1.4 & $6.2 \pm 0.3$ & $7.3 \pm 0.6$ \\
8 & Diamide & 41.6 & \pm 3.0 & $31.6 \pm 5.5$ & $47.0 \pm 4.4$ \\
\hline
\end{tabular}

9

10

11

12

13

14

15

16

17

18

19

20

21

22

23

24

25 
3 FIG. 1. (A) Biosynthetic pathway of ERG and (B) structure of MSH.

5 FIG. 2. In vitro assay of recombinant SCO0910 (EgtA). (A) Purified SCO0910 (EgtA) was

6 analyzed by SDS-PAGE. Lane 1, molecular mass markers; lane 2, purified maltose binding 7 protein-fused recombinant SCO0910 (89.6 kDa). (B) L-Glutamate and L-cysteine were 8 incubated in the presence of ATP without (upper) and with (lower) maltose binding 9 protein-fused recombinant SCO0910. The reaction product was analyzed by LC-ESI-MS. A 10 mass of 251, which correspond to $[\mathrm{M}+\mathrm{H}]^{+}$of $\gamma$-glutamyl-cysteine, was selected.

12 FIG. 3. Growth curves of the ERG-disruptant (circle), MSH-disruptant (square), and parental 13 strain (triangle). Growth was measured by dry cell weight. 


\section{$1 \quad$ Figure 1}

2

A
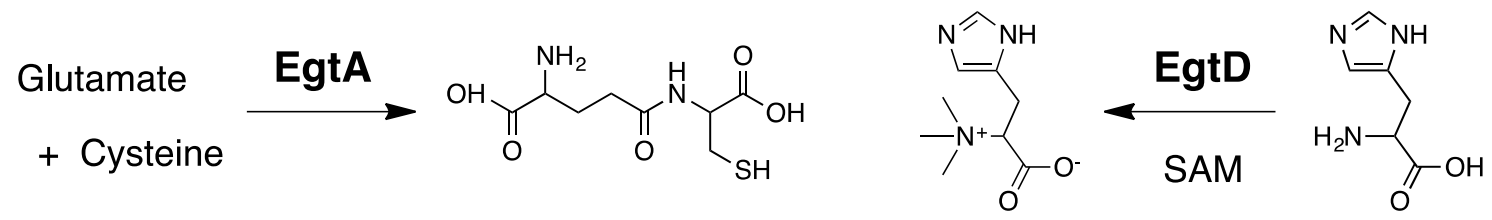

$\gamma$-Glutamyl-cysteine

Hercynine

Histidine

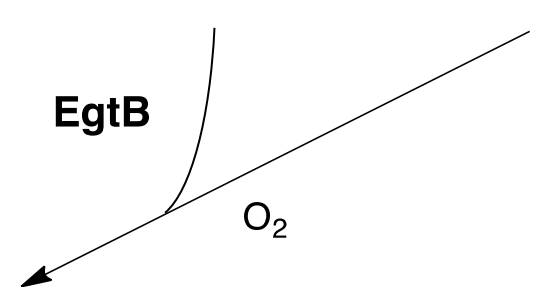

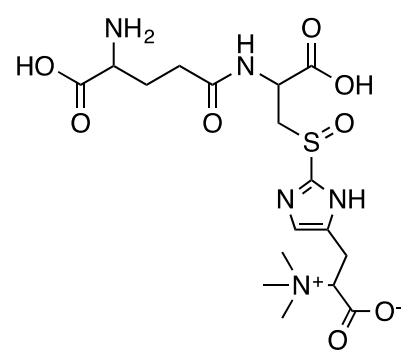

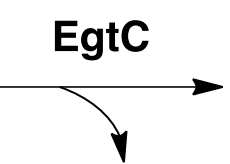

Glutamate

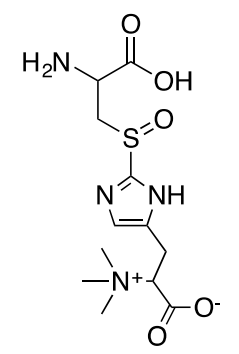

Hercynylcysteine sulfoxide

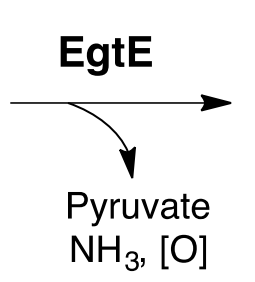<smiles></smiles>

Ergothioneine

B<smiles>NC(=O)C(CS)NC(=O)C(N)C1C(OC2C(O)C(O)C(O)C(O)C2O)OC(CO)C(O)C1O</smiles> 
$1 \quad$ Figure 2
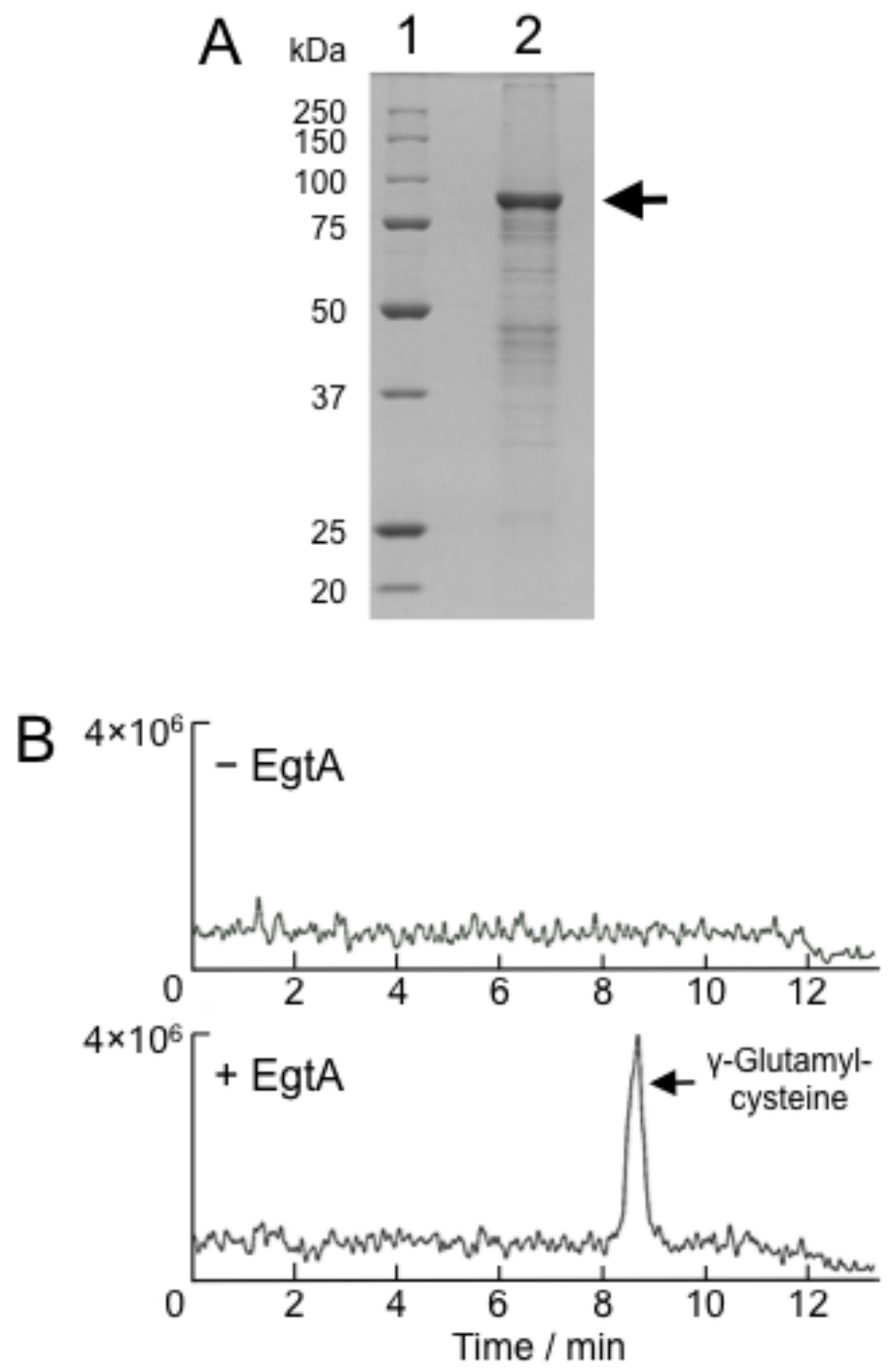
$1 \quad$ Figure 3

2

3

4

5

6

7

8

9

10

11

12

13

14

15

16

17

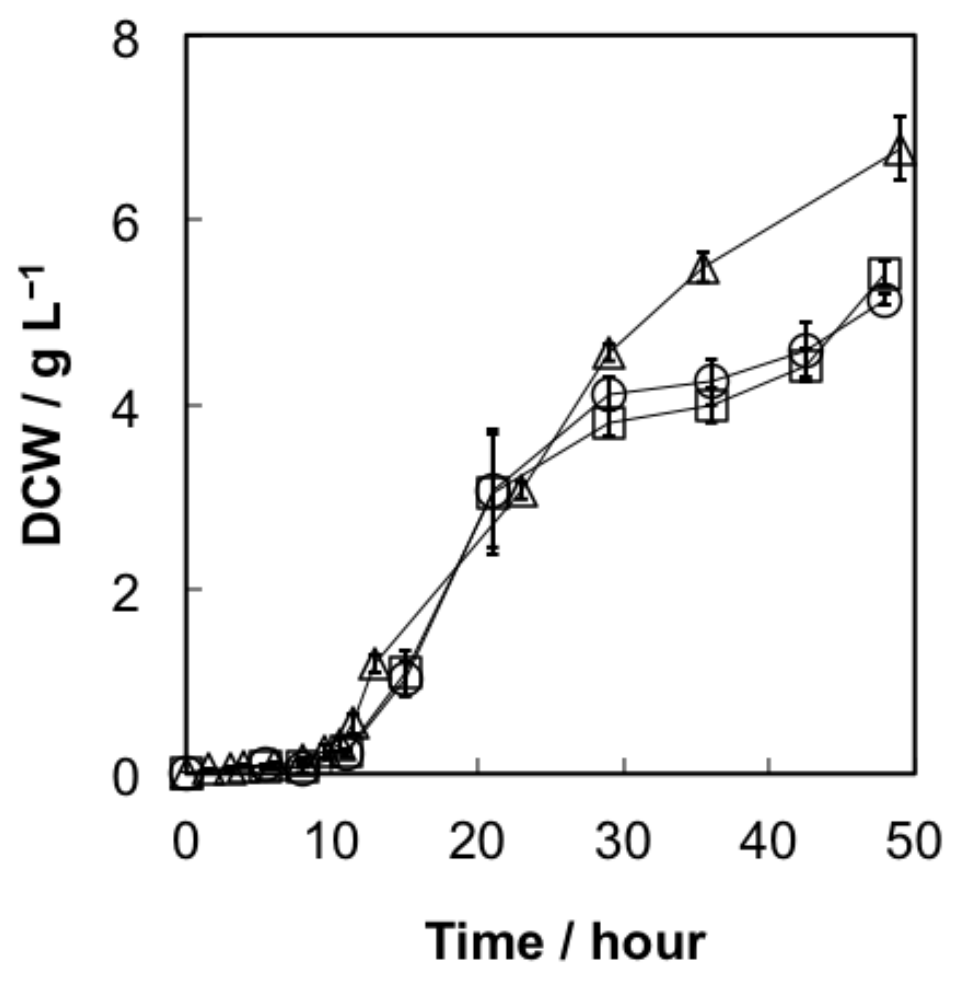

